\title{
BMJ Open Educational interventions for improving control of blood pressure in patients with hypertension: a systematic review protocol
}

\author{
Cibele D Ribeiro, ${ }^{1}$ Vanessa R Resqueti, ${ }^{1}$ íllia Lima, ${ }^{1}$ Fernando A L Dias, ${ }^{2}$ \\ Liam Glynn, ${ }^{3}$ Guilherme A F Fregonezi ${ }^{1}$
}

To cite: Ribeiro CD,

Resqueti VR, Lima Í, et al. Educational interventions for improving control of blood pressure in patients with hypertension: a systematic review protocol. BMJ Open 2015;5:e006583

doi:10.1136/bmjopen-2014006583

- Prepublication history for this paper is available online. To view these files please visit the journal online (http://dx.doi.org/10.1136/ bmjopen-2014-006583).

Received 10 September 2014 Revised 28 November 2014 Accepted 8 December 2014

CrossMark

\begin{abstract}
${ }^{1}$ Department of Physical Therapy, Federal University of Rio Grande do Norte, Natal, Rio Grande do Norte, Brazil ${ }^{2}$ Department of Physiology, Federal University of Paraná, Curitiba, Paraná, Brazil

${ }^{3}$ College of Medicine, Nursing and Health Sciences, National University of Ireland Galway, Galway, Ireland
\end{abstract}

\section{Correspondence to}

Professor Guilherme Augusto de Freitas Fregonezi; fregonezi.guilherme@gmail. com

\section{ABSTRACT \\ Introduction: The aim of this review is to evaluate the effectiveness of educational interventions on improving the control of blood pressure in patients with hypertension.}

Methods: Randomised controlled trials including patients over 18 years of age, regardless of sex and ethnicity, with a diagnosis of hypertension (either treated or not treated with antihypertensive medications) will be assessed in our analysis. We will electronically search four databases: MEDLINE, CINAHL, PEDro and ScienceDirect. There will be no language restrictions in the search for studies. The data will be extracted independently by two authors using predefined criteria. Disagreements will be resolved between the authors. The risk of bias will be assessed using the Cochrane risk of bias tool. After searching and screening of the studies, we will run a meta-analysis of the included randomised controlled trials. We will summarise the results as risk ratio for dichotomous data and mean differences for continuous data.

Ethics and dissemination: The review will be published in a journal. The findings from the review will also be disseminated electronically and at conference presentations.

Trial registration number: PROSPERO CRD4201401071.

\section{INTRODUCTION}

\section{Description of the condition}

Hypertension is a major health problem worldwide, and is estimated to cause more than $13 \%$ of deaths annually. ${ }^{1}$ It is a multifactorial clinical condition characterised by high and sustained levels of blood pressure. ${ }^{2}$ It is one of the most important public health problems in the world and an important modifiable risk factor for the development of cardiovascular diseases. Adoption of healthy lifestyles by all individuals is critical for the prevention of high blood pressure and is an

\section{Strengths and limitations of this study}

This systematic review will help:

- Clinicians to make decisions in clinical practice;

- Patients to better understand their conditions;

- To heighten awareness about disease progression and complication.

indispensable part of the management of those with hypertension. ${ }^{3}$ Uncontrolled hypertension is associated with high risk for development of heart disease, stroke, chronic kidney disease, retinopathy and peripheral vascular disease.

A recent epidemiological worldwide study estimated that high blood pressure causes approximately 7.6 million premature deaths (54\% for stroke and $47 \%$ for ischaemic heart disease). ${ }^{4}$ A recent systematic review reporting data from studies in 35 different countries between the years 2003 and 2008 demonstrated an overall prevalence of $37.8 \%$ for men and $32.1 \%$ for women. ${ }^{5}$

Owing to the fact that the prevalence of hypertension increases with age, ${ }^{6}$ the management of hypertension, and the prevention and treatment of major complications related to hypertension, will continue to be a global challenge for healthcare professionals.

\section{Description of the intervention}

Hypertension is a condition almost entirely managed by the primary care team including a variety of health professionals such as physicians, nurses, pharmacists and other allied healthcare professionals such as physiotherapists, who frequently work in cardiac rehabilitation. All professionals can potentially play an important role in lowering blood pressure. It is important that patients understand the benefits of blood pressure lowering, 
which include a reduction in many complications such as stroke $(35-40 \%)$, heart attack $(20-25 \%)$ and heart failure (over $50 \%$ ). ${ }^{3}$ The majority of patients will require a combination of antihypertensive drugs to reach target blood pressure.

A previous study demonstrated that educational interventions increased participants' levels of knowledge about hypertension and had a positive influence on their beliefs about medicines. ${ }^{7}$ Educational interventions can also create opportunities for patients to better understand their conditions and the role of therapies, as well as heighten awareness about disease progression and complications. Through patient education, misconceptions that patients have about their therapy can be clarified. This can influence adherence to therapy, ${ }^{8}$ and may therefore potentially lead to improved blood pressure control.

\section{How the intervention might work}

Different health professionals have become more involved in delivering interventions to their patients with the objective of preventing complications caused by high blood pressure. Patient expectations have a significant effect on the treatment patients get from their doctor or other health professional. ${ }^{9}$ Many previous trials in blood pressure control have used educational interventions on patients, physicians, or both, in an attempt to improve blood pressure control.

Educational interventions can positively modify patients' beliefs, which in turn can lead to a change in patient behaviour, such as improvement in adherence to a therapy proposed by the healthcare professional ${ }^{8}$ and a possible effect on variables related to the disease, such as blood pressure levels. This may also affect, in the long term, progression of the disease and the prevalence of associated conditions related to hypertension, such as heart attacks and stroke.

\section{Why it is important to present this review}

Owing to the high morbidity and mortality caused by hypertension, and the global scale of this important public health issue, it is important to continue to investigate interventions that can improve blood pressure control. It is striking that blood pressure goals continue to be achieved in only $25-40 \%$ of the patients who take antihypertensive drug treatment, ${ }^{3}{ }^{10}$ which is a statistic that has remained unchanged for the past 40 years. ${ }^{11}$

A recent Cochrane review demonstrated that there are many categories of interventions that singly or in unison have the potential to reduce blood pressure in patients with hypertension. ${ }^{12}$ In this review, educational interventions directed to patient and physician were examined; however, the focus of the review and protocol was not the educational intervention alone. Educational interventions, whether delivered to health professionals or patients, did not appear to be associated with large net reductions in blood pressure, but were recommended as an adjunct additional therapy along with other types of interventions.

Owing to the fact that there has been an increasing number of recent studies showing the importance of prevention in patients with hypertension, ${ }^{13-15}$ our review will determine the current evidence of the effects of educational interventions to improve control of blood pressure in patients with hypertension, potentially updating the recommendation for clinical practice.

\section{METHODS AND ANALYSIS}

\section{Criteria for considering studies for the review}

\section{Types of studies}

We plan to include randomised clinical trials (RCTs) that have evaluated the effects of different models of educational interventions with the overall aim of improving blood pressure control in patients with hypertension, irrespective of language. The review will include RCTs where educational interventions used as the main or adjunct treatment were compared to no educational interventions or different types of educational strategies. We will exclude studies that use educational interventions not intended to improve blood pressure control.

\section{Types of participants}

We will include studies that have participants over 18 years of age, regardless of sex and ethnicity, with a diagnosis of hypertension either treated or not treated with antihypertensive medications in a primary care, outpatient or community setting.

\section{Types of interventions}

The intervention of interest will include all educational intervention strategies designed to improve the control of blood pressure in patients with hypertension (eg, educational interventions direct to the patient; educational interventions direct to the health professional). Comparators will be any educational intervention used as the main or adjunct treatment to improve the control of blood pressure compared with either no educational interventions or different types of educational strategies aimed to improve blood pressure control.

\section{Types of outcome assessments}

The primary outcome of this review will be any changes in mean systolic blood pressure (SBP) and/or mean diastolic blood pressure (DBP) in any care setting as well as the number of patients under blood pressure (BP) control or proportion of controlled BP defined by each randomised trial's investigators. The secondary outcomes will be: number of hospitalisations during treatment (eg, increase of BP) or mortality from cardiovascular disease as adverse events; the costs and cost effectiveness of interventions; and the adherence to intervention (dropout rate) or adherence to medication; the outcome quality of life will be measured using standardised generic questionnaire. 
Search methods for identification of studies

Electronic searches

We will electronically search the following databases: MEDLINE, CINAHL, PEDro and ScienceDirect, without any language restrictions (in case of studies in a language other than English, we will contact companies specialised in translation). The search strategy will be developed after discussion among reviewers, according to the guidance of the Cochrane handbook. ${ }^{16}$ The MEDLINE search strategy will be translated into the other databases using appropriate controlled vocabulary as applicable for each database.

\section{Other sources}

The bibliographies of all retrieved and relevant publications identified by the above strategies will be searched for further studies. In addition, we will search the WHO International Clinical Trials Registry Platform (ICTRP; http://apps.who.int/trialsearch/). We will attempt to contact researchers to obtain additional information when needed.

\section{Data collection and analysis}

Selection of studies

Before the selection of studies, a procedure for screening will be developed by discussion among all the reviewers. We will extract data into Review Manager $5.3^{17}$ and summarise details using a standard data extraction sheet. Two reviewers (CR and VR) will independently assess the titles and abstracts of the studies identified from the search strategy against the inclusion criteria. Full versions of articles that appear to fulfil the inclusion criteria will be obtained for further assessment. Another review author (IL) will evaluate any discrepancies, if necessary, and will advise in case of disagreement. We will record all reasons for exclusion and exclude studies that do not use educational interventional to improve blood pressure control.

\section{Data extraction and management}

Two review authors (CR and VR), working independently, will extract data and summarise details of trials using a standard data extraction sheet. According to methods described in the Cochrane Handbook for Systematic Reviews of Interventions, ${ }^{18}$ the extraction sheet includes information such as study design, methodology, participants, interventions, duration of treatment, outcomes, conclusions and potential sources of bias. We will resolve any discrepancies by discussion with a third review author (IL). If studies report more than one outcome time (eg, 6 and 12 months), data concerning the longest follow-up will be extracted. Where data are found to be missing, we will contact the corresponding author of the study to request the missing data or to clarify study details.
Assessment of risk of bias in included studies

For assessment of study quality and reporting bias, two reviewers (CR and VR) will independently assess the risk of bias, using the Cochrane collaboration's tool for assessing risk of bias of the included trials, ${ }^{19}$ which is composed of six domains of a trial, such as random sequence generation (selection bias), allocation concealment (selection bias), blinding (performance bias and detection bias), incomplete outcome data (attrition bias), selective outcome reporting (reporting bias) and other bias. After assessing all the domains, the reviewers will summarise the assessments and categorise the included trials into three levels of bias: low, unclear and high risk of bias. We will resolve any disagreements by discussion with a third author (IL).

\section{Measures of treatment effect}

We will present the effects on blood pressure between interventions at follow-up (systolic and diastolic blood pressure) according to the educational interventions proposed in each study. We will present the outcome results for each trial with $95 \%$ CIs. Continuous outcomes (such as changes in systolic and diastolic blood pressure) will be expressed and calculated as mean difference (MD), and overall effect size between intervention and control groups. We will use Relative Risk (RR) or OR depending on measurements indices in individual studies for other primary and secondary outcomes.

\section{Dealing with missing data}

In the case of missing data, we will contact the original investigators to request missing data whenever possible. If the trial does not specify a participant group number prior to dropout, we will present only complete case analysis for primary and secondary outcomes.

\section{Assessment of heterogeneity}

Whenever studies appear to be similar in terms of participant characteristics (established hypertensive, people with diabetes or other chronic disease), intervention type, duration and outcome type, we will pool data using meta-analysis (using RevMan 5.3). We will test statistical heterogeneity using the $\chi^{2}$ test (considering a value of $\mathrm{p}<0.1$ to indicate heterogeneity) and estimate the amount of heterogeneity using the $\mathrm{I}^{2}$ statistic. ${ }^{20}$ If $\mathrm{I}^{2}$ is over $50 \%$, indicating a high level of heterogeneity, data will not be pooled. In the absence of clinical and statistical heterogeneity we will use a fixed-effect model.

\section{Assessment of reporting biases}

We will present the overall risk of bias (random sequence generation, allocation concealment, blinding of participants and personnel, blinding of outcome assessment, incomplete outcome data, selective reporting) in a risk of bias summary table per study. If sufficient studies (more than 10) are identified, an attempt will be made to examine for publication bias using a funnel plot, as described in the Cochrane Handbook for Systematic 
Reviews of Interventions. ${ }^{21}$ If asymmetry is present, we will explore possible causes including publication bias, poor methodological quality and true heterogeneity.

\section{Data synthesis}

We will present a narrative overview of the combined studies with meta-analysis of outcome data using the software Review Manager V.5.3 where appropriate.

We will include cluster-randomised trials in the analysis. For cluster-randomised trials, we will adjust results when the unit of analysis in the trial is presented as the total number of individual participants instead of number of clusters. Results will be adjusted using the mean cluster size and intracluster correlation coefficient. ${ }^{22}$ For meta-analysis, data will be combined to individually randomised trials using the generic inverse-variance method as described in Chapter 16.3 of the Cochrane Handbook for Systematic Reviews of Interventions. ${ }^{22}$

The decision to include studies in a meta-analysis will depend on the availability of treatment effect data and assessment of heterogeneity. Intervention effects will be calculated as RRs with $95 \%$ CIs for dichotomous data. For continuous data, we will calculate mean differences with $95 \%$ CIs using a conservative fixed-effects meta-analysis model in the absence of significant heterogeneity ( $p>0.05$ or $\mathrm{I}^{2}<50 \%$ ). If there is a high level of heterogeneity $\left(\mathrm{I}^{2}>50 \%\right)$, we will not pool data and we will perform sensitivity analysis of data.

\section{Subgroup analysis}

Subgroup analysis will be carried out according to the following variables: age and gender of participants, and the professional delivering the intervention (eg, nurse).

\section{Sensitivity analysis}

If sufficient trials are identified, we plan to conduct sensitivity analyses in order to explore the influence on the results of the following factor: assessor blinding (high risk of bias vs low risk of bias). We will restrict analyses to studies at low risk of bias.

\section{Ethics and dissemination}

This systematic review does not need ethical approval. Findings of this review will be disseminated via peerreviewed journals and conference presentations.

\section{DISCUSSION}

This is the protocol for a review and there is no primary data collection. The systematic review will be published in a peer-reviewed journal and disseminated electronically or in print. This review will also benefit patients with hypertension as they will better understand and accept the therapy, and change their behaviour regarding the treatment.

Contributors The search strategy will be developed and run by IL. Copies of studies will be obtained by CDR and VRR. Selection of the studies to include will be performed by CDR and VRR. Extraction data from studies and entering data into RevMan will be conducted by CDR and VRR. The analysis will be carried out by CDR, VRR and IL. Interpretation of the analysis will be carried out by all authors. The final review will be drafted by all authors. The protocol was revised, and the final version was approved, by all authors.

Funding The work was supported by the National Council for Scientific and Technological Development (CNPQ): 457357/2013-4 CTSaúde/Edital MCTI/ CNPQ/MS-SCTIE-DECIT n 57/2013 PEC-REBRATS—Brazilian Network for Health Technology Assessment in Health: Comparative Effectiveness Research.

Competing interests None.

Provenance and peer review Not commissioned; externally peer reviewed.

Open Access This is an Open Access article distributed in accordance with the Creative Commons Attribution Non Commercial (CC BY-NC 4.0) license, which permits others to distribute, remix, adapt, build upon this work noncommercially, and license their derivative works on different terms, provided the original work is properly cited and the use is non-commercial. See: http:// creativecommons.org/licenses/by-nc/4.0/

\section{REFERENCES}

1. World Health Organization (WHO). The world health report 2002 reducing risks, promoting healthy life. http://www.who.int/whr/2002/ en/ (accessed 1 Aug 2014).

2. Williams B. The year in hypertension. J Am Coll Cardiol 2008;51:1803-17.

3. Chobanian AV, Bakris GL, Black HR, et al. The seventh report of the joint national committee on prevention, detection, evaluation, and treatment of high blood pressure-the JCN 7 report. J Am Med Association 2003;289:2560-72.

4. Lopez AD, Mathers CD, Ezzati M, et al. Measuring the Global Burden of Disease and Risk Factors, 1990-2001. Global Burden of Disease and Risk Factors, 2006. http://www.dcp2.org/pubs/GBD (accessed 18 Sep 2012).

5. Pereira M, Lunet N, Azevedo A, et al. Diferences in prevalence, awareness, treatment and control of hypertension between developing and developed countries. J Hypertens 2009;27:963-75.

6. Kearney PM, Whelton M, Reynolds K, et al. Global burden of hypertension: analysis of worldwide data. Lancet 2005;365:217-23.

7. Magadza C, Radloff SE, Srinivas SC. The effect of an educational intervention on patients' knowledge about hypertension, beliefs about medicines, and adherence. Res Soc Adme Pharm 2009;5:363-75.

8. Saounatsou M, Patsi O, Fasoi G, et al. The influence of the hypertensive patient's in compliance with their medication. Public Health Nursing 2001;18:436-42.

9. McKinstry B, Hanley J, Heaney D, et al. Impact on hypertension control of a patient-held guideline: a randomised controlled trial. Br J Gen Pract 2006;56:842-7.

10. Burnier M. Blood pressure control and the implementation of guidelines in clinical practice: can we fill the gap? J Hypertens Supp 2002;20:1251-3.

11. Wilber J, Barrow J. Hypertension: a community problem. Am J Med 1972;52:653-63.

12. Glynn LG, Murphy AW, Smith SM, et al. Interventions used to improve control of blood pressure in patients with hypertension. Cochrane Database Syst Rev 2010;(3):CD005182.

13. Aung MN, Yuasa M, Moolphate $S$, et al. Reducing salt intake for prevention of cardiovascular diseases in high-risk patients by advanced health education intervention (RESIP-CVD study), Northern Thailand: study protocol for a cluster randomized trial. Trial 2012;13:158.

14. $\mathrm{Ng} \mathrm{N}$, Carlberg B, Weinehall $\mathrm{L}$, et al. Trends of blood pressure levels and management in Vasterbotten County, Sweden, during 1990-2010. Glob Health Action 2012;5:18195.

15. Ribeiro AG, Ribeiro SMR, Dias CMGC, et al. Non-pharmacological treatment of hypertension in primary health care: A comparative clinical trial of two education strategies in health and nutrition. BMC Public Health 2011;11:637.

16. Higgins JPT, Green S, eds. Cochrane handbook for systematic reviews of interventions version 5.1.0 [updated March 2011]. The Cochrane Collaboration, 2011. www.cochrane-handbook.org

17. Review Manager (RevMan) [Computer program]. Version 5.3. Copenhagen: The Nordic Cochrane Centre, The Cochrane Collaboration, 2014 
18. Higgins JPT, Deeks JJ. Chapter 7: selecting studies and collecting data. In: Higgins JPT, Green S, eds. Cochrane handbook for systematic reviews of interventions version 5.1.0 [updated March 2011]. The Cochrane Collaboration, 2011. http://www.cochrane-handbook.org

19. Higgins JPT, Altman DG, Sterne JAC. Chapter 8: assessing risk of bias in included studies. In: Higgins JPT, Green S, eds. Cochrane handbook for systematic reviews of interventions version 5.1.0 (updated March 2011). The Cochrane Collaboration, 2011. http:// www.cochrane-handbook.org

20. Deeks JJ, Higgins JPT, Altman DG. Chapter 9: analysing data and undertaking meta-analyses. In: Higgins JPT, Green S, eds.

Cochrane handbook for systematic reviews of interventions version
5.1.0 (updated March 2011). The Cochrane Collaboration, 2011. http://www.cochrane-handbook.org

21. Sterne JAC, Egger M, Moher D. Chapter 10: addressing reporting biases. In: Higgins JPT, Green S, eds. Cochrane handbook for systematic reviews of intervention version 5.1.0 (updated March 2011). The Cochrane Collaboration, 2011. http://www.cochranehandbook.org

22. Higgins JPT, Deeks JJ, Altman DG. Chapter 16: special topics in statistics. In: Higgins JPT, Green S, eds. Cochrane handbook for systematic reviews of interventions version 5.1.0 (updated March 2011). The Cochrane Collaboration, 2011. http://www. cochrane-handbook.org 